\title{
Clinical trial of bromhexine in severe chronic bronchitics during winter
}

\author{
S. W. CLARKE ${ }^{1}, G$. M. CRAIG, and E. J.B. MAKIN \\ Department of Medicine, University of Birmingham, Queen Elizabeth Hospital, Birmingham, B15 2TH \\ and Boehringer Ingelheim Limited, Isleworth, Middlesex
}

\begin{abstract}
In a double-blind, controlled cross-over trial, the effect of the mucolytic drug bromhexine ( $24 \mathrm{mg}$ or $48 \mathrm{mg}$ daily) was compared with a placebo over a period of 14 weeks in 21 severe chronic bronchitics (mean $\mathrm{FEV}_{1 \cdot 0} \mathbf{0 . 8}$ 1.). Each of the three treatment regimes was continued for four weeks with intervening single weeks on placebo tablets. As the subjects were outpatients, sodium fluorescein incorporated in the tablets was used as a drug marker. Satisfactory data were obtained in 18 patients. There was no significant improvement in clinical condition or ventilatory capacity nor change in sputum properties throughout the period of study. No side effects or significant change in blood values were detected.
\end{abstract}

Bromhexine is a benzylamine derivative of vasicine, an alkaloid derived from the plant Adhatoda vasica, which is indigenous to the continent of Asia. It has been shown to reduce the viscosity of bronchial secretions in animals (Engelhorn and Püschmann, 1963) and man (Hamilton, Palmer, and Gent, 1970) probably by depolymerization of high molecular weight mucopolysaccharide fibres (Boyd and Sheppard, 1966). Reduction of sputum viscosity should facilitate bronchial clearance in patients with diseases such as chronic bronchitis in which sputum may be copious, viscid, and difficult to expectorate. Since bromhexine is taken orally, its distribution and site of action within the lung should be uniform, thereby avoiding one of the major problems encountered in administering mucolytic agents by aerosol.

Previous studies of this drug in chronic bronchitis have not shown consistent results. Gent, Knowlson, and Prime (1969) found improvement

1Present address: Brompton Hospital, London S.W.3 in the clinical condition and ventilatory capacity of their patients, while others (Hamilton et al., 1970; Langlands, 1970) found no such effect. These trials were carried out over short periods of time (7 to 14 days) and in bronchitics of widely varying severity. The present trial was undertaken to assess the effect of the drug on the clinical condition, ventilatory capacity, and sputum properties of a group of severe bronchitics during several winter months (January to April 1969) when cough and sputum are most severe and any improvement should be most obvious.

\section{PATIENTS AND METHODS}

Twenty-one male outpatients, all of whom had severe obstructive chronic bronchitis (Medical Research Council, 1965), were admitted to the trial. Further details and pulmonary function data are summarized in Table I. They were selected because chronic cough, sputum, and difficulty in expectoration were troublesome symptoms; all were smokers. They were known to have good previous attendance records and to be

T A B L E I

DETAILS OF PATIENTS; MEAN VALUES AND STANDARD ERRORS (SEM) OF PULMONARY FUNCTION TESTS, PER CENT PREDICTED VALUES IN PARENTHESES 1 For the two ratios $\left(\mathrm{FEV}_{1.0} / \mathrm{FVC}\right.$ and $\left.\mathrm{RV} / \mathrm{TLC}\right)$ the mean values and ranges are shown.

\begin{tabular}{|c|c|c|c|c|c|c|c|}
\hline \multirow{2}{*}{$\begin{array}{c}\text { Mean Age } \\
\text { and Range (yr) }\end{array}$} & \multicolumn{7}{|c|}{ Pulmonary Function Tests } \\
\hline & FEV $_{1.0}(1)$. & FVC (1.) & $\mathrm{FEV}_{1.0} / \mathrm{FVC}(\%)$ & PFR (1/min) & RV (1.) & TLC (1.) & RV/TLC (\%) \\
\hline $63 \cdot 5(47-78)$ & $\begin{array}{c}0.80 \pm 0.06 \\
(25 \%)\end{array}$ & $\begin{array}{c}1 \cdot 47 \pm 0 \cdot 10 \\
(34 \%)\end{array}$ & $53(39-75)$ & $\begin{array}{c}165 \pm 10 \\
(34 \%)\end{array}$ & $\begin{array}{c}4 \cdot 22 \pm 0 \cdot 25 \\
(171 \%)\end{array}$ & $\begin{array}{c}7 \cdot 04 \pm 0 \cdot 31 \\
(100 \%)\end{array}$ & $60(40-68)$ \\
\hline
\end{tabular}

${ }^{1}$ Normal values were predicted from the formulae of Cotes, J. E. (1965), Lung Function, p. 341. Blackwell, Oxford. 
reliable in taking treatment. Those with cor pulmonale, severe incapacity or multiple drug therapy were excluded. All the patients had been followed for several years and there were previous clinical and pulmonary function data for comparison. The majority of them came from similar social backgrounds (Registrar General's class IV or V).

The trial was a double-blind, controlled cross-over study in which each patient received either $24 \mathrm{mg}$ or $48 \mathrm{mg}$ of bromhexine (Bisolvon) daily or identical placebo tablets in a sequence determined by a randomization scheme; with each regime the number of tablets was kept constant ( 2 three times a day) by the addition of placebo tablets to the lower dose regime of bromhexine. Two dose regimes of bromhexine were used because recent studies suggested that the effect of the drug may be dose-related (Hamilton et al., 1970). Since the trial was on outpatients, who may omit taking their tablets, a small amount $(5 \mathrm{mg})$ of sodium fluorescein was incorporated as a drug marker. This is excreted unchanged in the urine and imparts fluorescence under ultraviolet light.

Each period of treatment was for four weeks with intervening periods on placebo tablets of one week, the total duration of the trial being 14 weeks. The patients were instructed to continue long-standing treatment with sympathomimetic bronchodilators but to take no other tablets. Acute exacerbations were treated with a standard 10-day course of ampicillin. To reduce the risk of influenza, all patients were vaccinated during the first week of the trial.

The subjects attended the hospital weekly in three groups of seven. The randomization scheme was balanced so that a similar number of patients in each group was allocated to one of the three possible regimes. To facilitate hospital attendance, a weekly taxi service was organized to bring patients from their home or work. After being seen each patient was given an envelope containing the appropriate week's supply of tablets in individual daily dose packets with instructions for taking them.

Before the start of the trial each patient was assessed on the Medical Research Council short questionnaire. A special questionnaire designed to permit computeraided statistical analysis of the results was completed weekly at each patient's attendance by one of us (S.W.C. or G.M.C.). This covered symptoms (cough, wheezing, exercise dyspnoea, and general well-being), physical signs (breath and adventitial sounds, oedema, and cyanosis) and sputum (volume and pouring characteristics). The early morning sputum volume (that expectorated in the first hour after waking) was recorded daily by the patient at home using a standard plastic cup provided with a paper graduation. Its pouring characteristics were measured crudely by tipping the cup horizontal and recording the time taken for the sputum to flow out of the cup. On the day of attendance, the measurements of the early morning sputum samples were made by the hospital staff.

At weekly intervals the forced expired volume in 1 second $\left(F E V_{1.0}\right)$ and forced vital capacity (FVC) were measured by spirometry and the ratio $\mathrm{FEV}_{1.0} / \mathrm{FVC}$ was calculated in per cent. Peak flow rate (PFR) was measured with a Wright peak-flow meter. Lung volumes and their subdivisions were measured with a closedcircuit helium dilution technique at the beginning and end of each treatment regime (i.e., weeks $0,4,5,9,10$, and 14).

Blood was taken weekly for haemoglobin, white cell count and differential, serum albumin, globulin, bilirubin, and enzymes (aspartate aminotransferase, 5 nucleotidase, and alkaline phosphatase). Urine was tested for fluorescence.

Full weather details, including ambient temperature, barometric pressure and humidity, were obtained weekly from the local meteorological station to determine any correlation between change in weather conditions and clinical status. This seemed relevant since the trial was conducted in the winter and spring months.

\section{RESULTS}

All but three patients completed the trial satisfactorily, leaving 18 for analysis. Three patients had a single exacerbation of chronic bronchitis which settled within 10 to 14 days. These exacerbations were equally spaced throughout the trial and were not related to any particular regime. The detection of urinary fluorescence confirmed that every patient had taken his tablets.

Analysis of the data showed no uniform significant changes, either in symptoms, signs, sputum or pulmonary function, during the trial period (Tables II and III). There were no trends corresponding to the prevailing weather conditions. There were no side effects from bromhexine therapy and no significant alteration in the blood count, serum enzymes, serum proteins or bilirubin.

FOLLOW-UP The patients were assessed again two years later, when they were asked specifically for comments on their progress during the clinical trial. At this time two patients had died and one could not be traced, leaving 15 who were interviewed. Six patients thought they were improved generally during the trial, three thought their respiratory symptoms were reduced, and seven noted no change. Of the three patients who noted respiratory improvement, all thought their sputum was thinner and more easily cleared. These comments must be viewed in the light of the time that had elapsed since the trial, the failure of the cross-over to show significant benefit from the active drug, and the tendency of patients in clinical trials to show symptomatic improvement. This last point was demonstrated by a trend towards improvement during the trial, irrespective of the treatment order. 
T A B L E I I

SYMPTOMATIC ASSESSMENT OF PATIENTS ON PLACEBO, LOW DOSE (8 $\mathrm{mg}$ tds) OR HIGH DOSE (16 mg tds) REGIME, FROM THE SPECIAL QUESTIONNAIRE

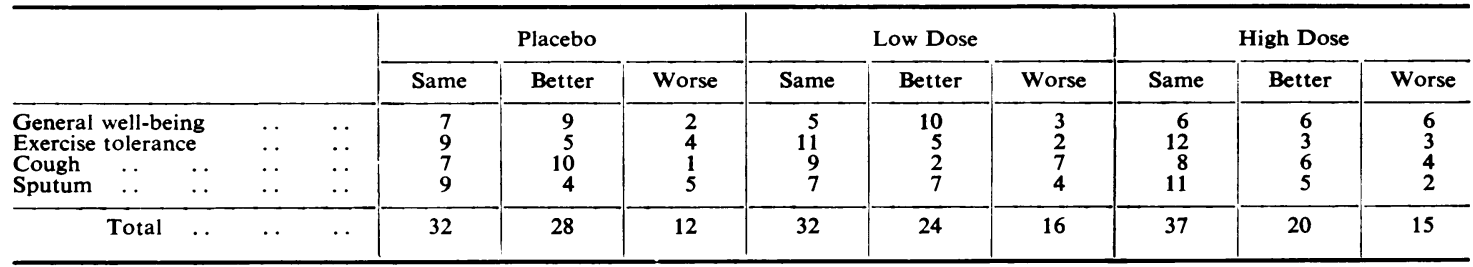

T A B L E I I I

MEAN RESULTS OF PULMONARY FUNCTION TESTS ( \pm SEM) WITH PATIENTS ON PLACEBO, LOW DOSE (8 mg tds) OR HIGH DOSE (16 mg tds) REGIME

\begin{tabular}{|c|c|c|c|c|c|c|c|}
\hline Test & & Control & Placebo & Control & Low Dose & Control & High Dose \\
\hline 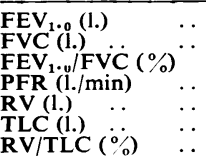 & $\begin{array}{l}\cdots \\
\cdots \\
\cdots \\
\cdots \\
\cdots \\
\cdots\end{array}$ & $\begin{array}{c}0 \cdot 80 \pm 0.06 \\
1 \cdot 47 \pm 0 \cdot 10 \\
55 \cdot 2 \\
165 \pm 10 \\
4 \cdot 22 \pm 0 \cdot 25 \\
7 \cdot 04 \pm 0 \cdot 31 \\
59 \cdot 4\end{array}$ & $\begin{array}{c}0.78 \pm 0.03 \\
1 \cdot 36 \pm 0.06 \\
58 \cdot 7 \\
146 \pm 5 \\
4 \cdot 13 \pm 0.26 \\
7 \cdot 10 \pm 0.32 \\
57 \cdot 7\end{array}$ & $\begin{array}{c}0 \cdot 74 \pm 0 \cdot 07 \\
1 \cdot 28 \pm 0 \cdot 12 \\
58 \cdot 1 \\
147 \pm 7 \\
4 \cdot 34 \pm 0 \cdot 24 \\
7 \cdot 23 \pm 0 \cdot 29 \\
59 \cdot 9\end{array}$ & $\begin{array}{c}0.75 \pm 0.03 \\
1 \cdot 35 \pm 0.06 \\
56.5 \\
141 \pm 5 \\
4.12 \pm 0.24 \\
6.96 \pm 0.30 \\
58.8\end{array}$ & $\begin{array}{c}0.75 \pm 0 \cdot 06 \\
1 \cdot 25 \pm 0 \cdot 10 \\
60 \cdot 9 \\
135 \pm 8 \\
4 \cdot 17 \pm 0 \cdot 24 \\
7 \cdot 10 \pm 0 \cdot 30 \\
58 \cdot 3\end{array}$ & $\begin{array}{c}0.77 \pm 0.03 \\
1.39 \pm 0.06 \\
56.6 \\
157 \pm 6 \\
4 \cdot 24 \pm 0.27 \\
7 \cdot 13 \pm 0.33 \\
59.0\end{array}$ \\
\hline
\end{tabular}

Comparison of the ventilatory tests in 1969 and the present showed the expected deterioration.

\section{DISCUSSION}

The results of the present double-blind crossover trial of bromhexine in doses of $24 \mathrm{mg}$ or $48 \mathrm{mg}$ daily in patients with chronic bronchitis show no significant effects of the drug on symptoms, signs or pulmonary function. These patients had severe airways obstruction as evaluated by a mean $\mathrm{FEV}_{1.0}$ of $0.8 \mathrm{l}$. In a group of 13 bronchitics of similar severity (mean $\mathrm{FEV}_{1.0} 0.71$.), treated with bromhexine, $24 \mathrm{mg}$ daily for 14 days in hospital, Langlands (1970) also found no drug effect, and Hamilton et al. (1970), treating a further group of 12 severe bronchitics (mean $\mathrm{FEV}_{1 \cdot 0} 28.8 \%$ predicted) with $48 \mathrm{mg}$ daily for 11 days, also found no improvement in ventilatory capacity or overall respiratory state. In contrast, Gent et al. (1969) found significant improvement in their mixed group of 23 patients treated with bromhexine, 24 mg daily for one week; this improvement was seen only in the winter months, and the patients were much less severely disabled than those in the present study, those with chronic bronchitis or asthma having a mean FEV F $^{\circ}$ of 2.41 . Christensen et al. (1971), in a long-term study over two winters, noted clinical improvement principally in moderate bronchitics (mean FEV . $_{1.0}$ 1.45 1.) but not in those with severe disease (mean $\mathrm{FEV}_{1 \cdot 0} 0.9$ 1.). Thus the results of the present study agree with these previous findings in severe bronchitics and suggest that patients with $\mathrm{FEV}_{1} \cdot 0$ of less than 1.01 . are unlikely to be improved by bromhexine. In these patients it is probable that the effects of irreversible structural change within the bronchi and lung parenchyma greatly exceed those of sputum in the airways. Although sputum may increase airway resistance (Clarke, Jones, and Oliver, 1970), its effect on the overall ventilatory capacity of bronchitics is largely unknown.

Examination of the sputum data showed the expected variation between patients but also differences for the same patient over the period of study. This may reflect the difficulties of collecting and measuring sputum encountered by the average bronchitic at home. The volume of sputum will depend on the efficiency of the cough mechanism in clearing the bronchial tree as well as the addition of saliva. Severe bronchitics, whose airways are abnormally collapsible, have an inefficient cough mechanism (Langlands, 1967) so that expectorated sputum may not closely reflect sputum production. In these patients the inefficiency of their cough mechanism is likely to be the major factor determining bronchial clearance, rather than the viscosity of sputum. Furthermore, as sputum is probably thixotropic, the physical agitation of expectoration will alter its viscosity, as will any delay in carrying out the measurement of pouring time (Sturgess, Palfrey, and Reid, 1970). These factors could account for the variation in viscosity measurements. 
In clinical trials on outpatients, confirmation that the drug is being taken is important and previous methods have proved unreliable. The method of incorporating a small amount of sodium fluorescein into the tablets has recently been described by Thompson and Stowers (1971) who found fluorescein to be a satisfactory drug marker. This provided a simple, innocuous but effective way of checking whether the patient was taking his tablets by the detection of urinary fluorescence.

While the results of this trial show no benefit from bromhexine in severe bronchitics, previous trials suggest that patients at a less advanced stage are improved. If bromhexine enhances bronchial clearance in these less advanced cases, the inevitable clinical deterioration of the chronic bronchitic may be slowed or arrested. This last point still awaits further clinical proof.

We thank the patients and technicians who co-operated in this study, Professor J. M. Bishop for his advice, Boehringer Ingelheim Limited for generous help with the trial, Mrs. P. Pollard for the statistical analysis, and Professor O. L. Wade for reading the manuscript.

\section{REFERENCES}

Boyd, E. M., and Sheppard, P. (1966). On the expectorant activity of Bisolvon. Arch. int. Pharmacodyn, 163, 284.

Christensen, S. B., Kjer, J., Ryskjaer, S., Årseth-Hansen, P., and Christensen, F. (1971). Mucolytic treatment of chronic bronchitis during two winter periods. Scand. $J$. resp. Dis., 52, 48.

Clarke, S. W., Jones, J. G., and Oliver, D. R. (1970). Resistance to two-phase gas-liquid flow in airways. $J$. appl. Physiol., 29, 464.

Engelhorn, R., and Püschmann, S. (1963). Pharmakologische Untersuchungen über eine Substanz mit sekretolytischer Wirkung. Arzneimittel-Forsch., 13, 474.

Gent, M., Knowlson, P. A., and Prime, F. J. (1969). Effect of bromhexine on ventilatory capacity in patients with a variety of chest diseases. Lancet, 2, 1094.

Hamilton, W. F. D., Palmer, K. N. V., and Gent, M. (1970). Expectorant action of bromhexine in chronic obstructive bronchitis. Brit. med. J., 3, 260.

Langlands, J. (1967). The dynamics of cough in health and in chronic bronchitis. Thorax, 22, 88.

- (1970). Double-blind clinical trial of bromhexine as a mucolytic drug in chronic bronchitis. Lancet, 1, 448.

Medical Research Council (1965). Definition and classification of chronic bronchitis for clinical and epidemiological purposes. Lancet, 1, 775.

Sturgess, J. M., Palfrey, A. J., and Reid, L. (1970). The viscosity of bronchial secretion. Clin. Sci., 38, 145.

Thompson, R. H., and Stowers, J. M. (1971). The use of a drug marker in a clinical trial. Scot. med. J., 16, 259. 